\title{
Comparison of theory with experiment for positron production from high-energy electrons moving along crystal axes
}

\author{
V. N. Baier and V. M. Strakhovenko \\ Budker Institute of Nuclear Physics, 630090 Novosibirsk, Russia
}

(Received 25 September 2002; published 5 December 2002)

\begin{abstract}
Using the approach developed earlier for the description of the electron-photon showers in axially aligned single crystals, some characteristics of the positron yield measured in recent experiments are calculated. Theoretical estimations display a rather good agreement with experimental results obtained using 3 to $10 \mathrm{GeV}$ electrons aligned to the $\langle 111\rangle$ axis of the tungsten crystals. Such comparison verified that the accuracy of our approach is quite sufficient to make a reliable choice for optimal parameters of the positron source using axially aligned crystals for future linear colliders.
\end{abstract}

DOI: 10.1103/PhysRevSTAB.5.121001

PACS numbers: 12.20.Ds, 03.65.Sq

\section{INTRODUCTION}

An efficient positron source is one of the important components of future electron-positron colliders. Positrons are generated from electrons in the course of the $e^{-} e^{+} \gamma$ shower developing in a medium. In a highenergy region, the basic processes involved in the shower development are typically considerably enhanced in oriented crystals as compared with corresponding amorphous media. The most pronounced effects take place at axial alignment when initial electrons are moving along the main axes of a crystal. This alignment alone will be considered below. According to [1], the radiation intensity in a crystal exceeds that of the conventional bremsstrahlung starting with electron energies $\varepsilon \sim 1 \mathrm{GeV}$. Simple estimations of the width of the power spectrum indicated a soft character of this spectrum. So the use of highenergy electrons impinging on the axially oriented crystals ".. as a source of hard and directed radiation concentrated within a comparatively narrow frequency range ..." was proposed in [1]. Based on mentioned properties of the photon emission process, the use of this phenomenon in the positron source for future accelerators was proposed [2,3]. The pair production rate which is due to the coherent (crystal) effects exceeds that of the standard (Bethe-Heitler) mechanism starting with photon energies $\omega \simeq \omega_{t h}$. The value of $\omega_{t h}$ is about $22 \mathrm{GeV}$ for the $\langle 111\rangle$ axis of tungsten being several times larger for other crystals. (See the review [4] and the recent book [5] for further details concerning QED processes in crystals.) For energies well above $\omega_{t h}$, the crystal effects become really strong and may be used to create effective and compact electromagnetic calorimeters [6]. For very high energies $\left(\varepsilon \gg \omega_{t h}\right)$ of initial and created particles, kinetic equations describing the shower development were solved analytically [7]. Though the initial electron energies were high enough in the first experimental investigation [8] of shower formation in crystals, energies of detected particles were too low to allow us the direct comparison with [7]. To explain the results of [8], Monte Carlo simulations were performed in [9]. The probabilities of basic processes used in [9] were obtained within so-called constant field approximation. A good agreement was demonstrated in [9] with the results of [8] for Ge crystals.

When the initial electron energy is below $\omega_{t h}$, photons are mainly emitted with energies $\omega \ll \omega_{t h}$ and so, up to minor modifications (see $[10,11]$ ), the pair production process proceeds in a crystal as in an amorphous medium. The enhancement of radiation from initial electrons is thereby the main crystal effect in this energy region. The substantial advance in the description of shower formation at axial alignment was caused by the invention of the semiphenomenological radiation spectrum [12]. This allows one to consider the relatively low (of a few $\mathrm{GeV}$ ) energy range of the initial electrons which is presumed for the efficient positron source. The radiation intensity increases with the initial electron energy. As a result, at some energy the effective radiation length $L_{\mathrm{ef}}$ in the crystal becomes smaller than the conventional radiation length $L_{\text {rad }}$ and continues its decrease at further increase of the energy. All numerical examples will be given below for the electron beam aligned with the $\langle 111\rangle$ axis of the tungsten crystals. Then we have for the quantity $L_{\mathrm{ef}}$ defined as in Sec. 3 of [12]: $L_{\mathrm{ef}}(1 \mathrm{Gev}) \simeq 0.166 \mathrm{~cm}$, $L_{\mathrm{ef}}(4 \mathrm{Gev}) \simeq 0.084 \mathrm{~cm}$, and $L_{\mathrm{ef}}(8 \mathrm{Gev}) \simeq 0.061 \mathrm{~cm}$. In the hybrid target which consists of the crystal part followed by the amorphous one, the thickness of the crystal constituent of several $L_{\text {ef }}$ is obviously quite enough. Indeed, at the depth $L_{0} \approx(3-4) L_{\mathrm{ef}}$ most of the particles, including the initial electrons, are sufficiently soft to reduce the coherent contribution to the radiation to the level of the incoherent one. Thereby, the further development of the shower proceeds more or less in the same way for the crystal or amorphous type of the remaining part of the target. We emphasize that the crystal part $L \leq L_{0}$ of the target serves as the radiator, and secondary charged particles are still not so numerous at this stage of the shower development. Therefore only a small portion of the total energy loss is deposited in the crystal part of the target which considerably reduces a danger of its 
overheating. The softness of the photon spectra is another important feature of the crystal radiator giving additional advantages for the positron production in comparison with the entirely amorphous target. To get a more definite idea concerning the shape of the power spectrum one can use its explicit form given by Eq. (2) in [12]. To present the scale, let us list some values $\omega_{\max }$ where this spectrum is maximum: $\omega_{\max }(1 \mathrm{GeV}) \simeq 31 \mathrm{MeV}, \omega_{\max }(4 \mathrm{GeV}) \simeq$ $170 \mathrm{MeV}$, and $\omega_{\max }(8 \mathrm{GeV}) \simeq 490 \mathrm{MeV}$. Note that the width of the spectrum is typically several times larger than $\omega_{\max }$. The increase in the number of relatively soft photons turns out to be much more pronounced than that in the total radiation intensity. In the end, just this fact leads to the substantial enhancement of the positron yield from crystal targets.

Recently the positron production in axially aligned single crystals was studied in two series of experiments performed at CERN $[13,14]$ and KEK $[15,16]$. The initial energy of electrons was $3 \mathrm{GeV}$ [15], 6 and $10 \mathrm{GeV}$ [14], $8 \mathrm{GeV}$ [16], and $10 \mathrm{GeV}$ [13]. In all cases the initial electron beam was aligned with the $\langle 111\rangle$ axis of the tungsten crystal that sometimes served as the crystal part of the hybrid target which contained an additional amorphous tungsten target. A noticeable enhancement of the low-energy positron yield was observed in all experiments cited above when the yield from the crystal target was compared with that from the amorphous target of the same thickness. The experimental results and our theoretical estimations presented in the next section display a rather good agreement with each other.

\section{COMPARISON OF THEORY WITH EXPERIMENT}

Theoretical results for the conditions of the experiments cited above were obtained using the approach developed in $[11,12]$ where various positron and photon distributions as well as deposited energies in different crystals were calculated for the energy range of initial electrons from 2 to $300 \mathrm{GeV}$. In these papers, all the formulas used in Monte Carlo simulations of the specific $e^{-} e^{+} \gamma$-shower characteristics are given in the explicit form. Remember that our simplified description of the shower development takes into account coherent induced by the regular motion of particles in the field of crystal axes) and incoherent like that in an amorphous medium) mechanisms of photon emission and pair production processes. The multiple scattering and the ionization energy loss of electrons and positrons are taken into account neglecting crystal effects. The coherent radiation from channeling and moving not very high above the axis potential barrier particles is described using the semiphenomenological spectrum suggested in [12]. The corresponding computer code was developed. This allows one to calculate energy, angular, and coordinate distributions of positrons emergent from the crystal or hybrid target and to find an amount of the energy deposition. We
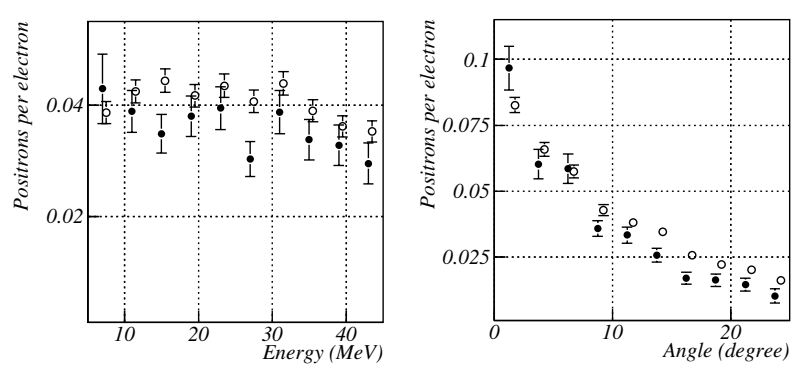

FIG. 1. Spectral (top) and angular (bottom) distributions of positrons from $10 \mathrm{GeV}$ electrons traversing a 8-mm-thick crystal tungsten target along the $\langle 111\rangle$ axis. Open circles: simulation; filled circles: experiment.

think that the investigation of such distributions should be the main object of the experiments having the creation of the crystal assisted positron source as their ultimate aim.

\section{A. Experiment (CERN) at $\varepsilon_{0}=10 \mathrm{GeV}$}

Among experiments cited above, spectral-angular distributions of created positrons were measured only in the WA103 experiment at CERN (see $[13,14]$ ), where our code was used in simulations as the event generator. This simulation allowed for the acceptance conditions and the efficiency of the detectors used. Shown in Fig. 1 taken from [14] is one example of the measured and simulated distributions of positrons from $10 \mathrm{GeV}$ electrons aligned with the $\langle 111\rangle$ axis of the $8 \mathrm{~mm}$-thick crystal tungsten.

The angular acceptance conditions in the WA103 experiment were approximately $\left|\vartheta_{V}^{\text {out }}\right| \leq 1.5^{\circ}$ for the vertical and $0 \leq \vartheta_{H}^{\text {out }} \leq 25^{\circ}$ for the horizontal angle of the outgoing positron with respect to the initial electron beam direction. We shall see below that the shape of the positron spectrum depends on the degree of collimation. The one-dimensional (over $\vartheta_{H}^{\text {out }}$ ) angular distribution is presented for positrons having energies in the 5-45 MeV range. We emphasize that the relative difference between measured and simulated results typically does not exceed $20 \%$ in both spectral and angular distributions as seen in Fig. 1. We are aware that preliminary results for other settings used in the same experiment do not contradict with the estimated scale of the difference between the data and theoretical predictions. We hope that this interrelation will not become worse after performing the complete analysis of the data which now is underway. This analysis will also give more detailed information concerning spectral-angular distributions of positrons depending on initial electron energies and target thicknesses.

\section{B. Experiment (KEK) at $\varepsilon_{0}=3 \mathrm{GeV}$}

The main goal of the experiment [15] was an attempt to apply the crystal target to the working electron/positron 
linac, the injector for the electron-positron collider BFactory at KEK. Thus, the acceptance conditions for created positrons were determined by the momentum acceptance of the positron linac with the matching section which is $8.2<p<11.6 \mathrm{MeV} / c$ and $p_{\perp}<$ $2.4 \mathrm{MeV} / c$. The hybrid target used consists of $1.7-\mathrm{mm}$ thick tungsten crystal followed by 7-mm-thick amorphous tungsten. The observed positron yield was enhanced by the factor 1.40 when the $\langle 111\rangle$ crystal axis was aligned with $3 \mathrm{GeV}$ incident electron beam as compared to the case of the disoriented crystal. Our number for this enhancement is 1.47 being only 5\% larger than the experimental one. Note that in the experiment [15] the crystal and amorphous parts of the hybrid target were separated by the distance of $70 \mathrm{~mm}$. This circumstance, which, in principle, may slightly change the enhancement value, was not taken into account in our calculation. Recollect that the amount of the energy deposited in the crystal part ( $\varepsilon_{\mathrm{dep}}^{\mathrm{cr}}$ ) of the hybrid target may be much smaller than that $\left(\varepsilon_{\text {dep }}^{\mathrm{am}}\right)$ in the amorphous one. Such interrelation of $\varepsilon_{\mathrm{dep}}^{\mathrm{cr}}$ and $\varepsilon_{\mathrm{dep}}^{\mathrm{am}}$ should take place in the case of [15], where the crystal thickness is about $1.8 L_{\text {ef }}$ (see the discussion in the Introduction). This is confirmed by our calculations which give $\varepsilon_{\mathrm{dep}}^{\mathrm{cr}} \simeq 11 \mathrm{MeV}$ and $\varepsilon_{\mathrm{dep}}^{\mathrm{am}} \simeq$ $277 \mathrm{MeV}$ per one incident electron.

\section{Qualitative features of positron distributions and experiment (KEK) at $\varepsilon_{0}=8 \mathrm{GeV}$}

In [16] the positron production efficiency from 2.2, 5.3, and $9.0-\mathrm{mm}$-thick tungsten crystals was measured using an $8-\mathrm{GeV}$ electron beam. Positrons produced in the forward direction with momenta 10,15 , and $20 \mathrm{MeV} / c$ were detected by the magnetic spectrometer. Thus, several points in the energy distribution were determined under hard collimation conditions. To give an idea of spectralangular distributions on the whole, let us remind their important qualitative features using $8 \mathrm{GeV}$ electrons and the $\langle 111\rangle$ axis of the tungsten crystals as an example. For the sake of comparison, the corresponding distributions for amorphous tungsten will be presented as well. Below all the quantities characterizing the positron yield are normalized per one incident electron.

The use of matching systems implies some collimation (typically $\vartheta_{\text {out }} \leq 25^{\circ}$ ) of outgoing positrons. Shown in Fig. 2 is the energy dependence (energy step is equal to $10 \mathrm{MeV}$ ) of the positron yield from crystal (a) and amorphous (b) targets of the same thickness $L=2.2 \mathrm{~mm}$. In the case of the hard collimation, when $\vartheta_{\text {out }} \leq 1^{\circ}$ (open circles), the yield is multiplied by 10 to make it visible. The larger the positron energy, the smaller the typical value of $\vartheta_{\text {out }}$ since both production and multiple scattering processes are characterized by smaller angles for higher energies. This is seen in Fig. 2(a) where the spectral curves for $\vartheta_{\text {out }}<180^{\circ}$ and that for $\vartheta_{\text {out }} \leq 24^{\circ}$ are overlapping within precision better than $1 \%$ starting from $\varepsilon_{\mathrm{cr}}^{(1)} \simeq 55 \mathrm{MeV}$. In turn, from $\varepsilon_{\mathrm{cr}}^{(2)} \simeq 110 \mathrm{MeV}$ the same happens with curves corresponding to $\vartheta_{\text {out }} \leq 24^{\circ}$ and $\vartheta_{\text {out }} \leq 12^{\circ}$. Such behavior is also seen in Fig. 2(b) for the amorphous target where $\varepsilon_{\mathrm{am}}^{(1)} \simeq 50 \mathrm{MeV}$ and $\varepsilon_{\mathrm{am}}^{(2)} \simeq$ $105 \mathrm{MeV}$.

In other words, positrons with energies $\varepsilon>\varepsilon^{(1)}$ are practically concentrated within the cone $\vartheta_{\text {out }} \leq 24^{\circ}$ and those with $\varepsilon>\varepsilon^{(2)}$ have $\vartheta_{\text {out }} \leq 12^{\circ}$. In accordance with this picture, the spectral maximum is shifted to the right while the width of the distribution increases when the collimation angle decreases. The enhancement $\mu$, being a bin-by-bin ratio of the positron yield from the crystal target to that from the amorphous one at the same collimation, is almost constant for $\varepsilon<45 \mathrm{MeV}$ and monotonically decreases with growing positron energy. This means that positron spectra from the crystal target are softer. Somewhat lower values of $\varepsilon^{(1)}, \varepsilon^{(2)}$ in the amorphous case point at the same feature. For given collimation, the variation of the enhancement is about $20 \%$ over the whole energy interval presented in Fig. 2. The maximum values of the enhancement at different collimation are $\mu_{\text {max }}\left(\vartheta_{\text {out }} \leq 180^{\circ}\right) \simeq 6.09, \mu_{\text {max }}\left(\vartheta_{\text {out }} \leq 24^{\circ}\right) \simeq 5.92$,
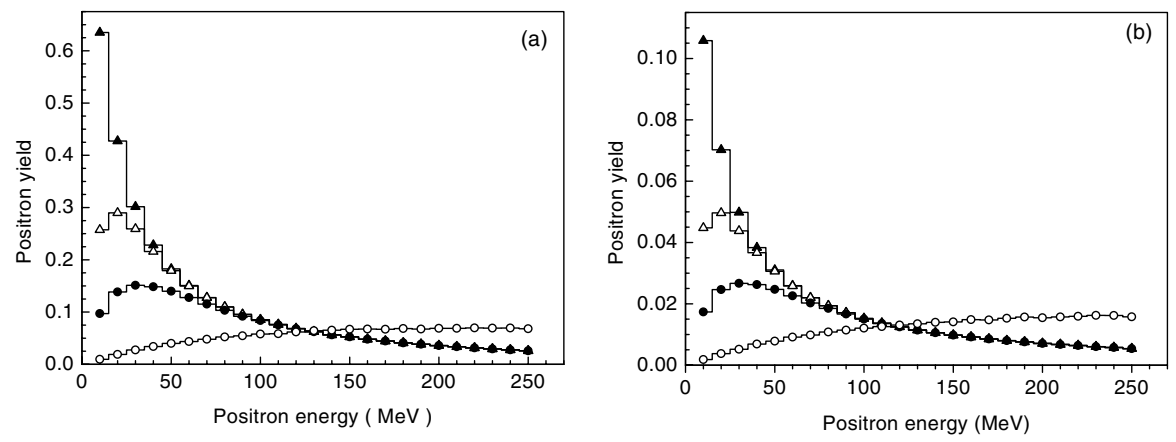

FIG. 2. Positron yield depending on energy from 2.2-mm-thick crystal (a) and amorphous (b) targets at different collimation. Filled triangles: no collimation $\left(\vartheta_{\text {out }} \leq 180^{\circ}\right)$; open triangles: $\vartheta_{\text {out }} \leq 24^{\circ}$; filled circles: $\vartheta_{\text {out }} \leq 12^{\circ}$; and open circles: $\vartheta_{\text {out }} \leq 1^{\circ}$ (multiplied by 10$)$. 

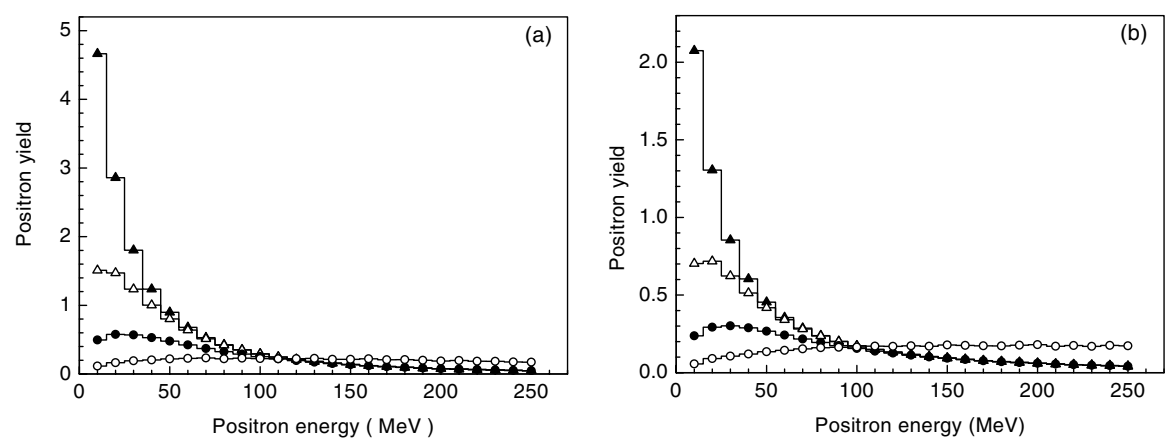

FIG. 3. Positron yield depending on energy from 9.0-mm-thick crystal (a) and amorphous (b) targets at different collimation. Filled triangles: no collimation $\left(\vartheta_{\text {out }} \leq 180^{\circ}\right)$; open triangles: $\vartheta_{\text {out }} \leq 24^{\circ}$; filled circles: $\vartheta_{\text {out }} \leq 12^{\circ}$; and open circles: $\vartheta_{\text {out }} \leq 1^{\circ}$ (multiplied by 30$)$.

$\mu_{\max }\left(\vartheta_{\text {out }} \leq 12^{\circ}\right) \simeq 5.67$, and $\mu_{\max }\left(\vartheta_{\text {out }} \leq 1^{\circ}\right) \simeq 5.29$. Apparently, they diminish as the collimation angle does so.

Figure 3 is the same as Fig. 2 but for the target thickness $L=9.0 \mathrm{~mm}$. The yield at $\vartheta_{\text {out }} \leq 1^{\circ}$ (open circles) is multiplied now by 30 . The qualitative behavior of spectra depending on the collimation angle at $L=9.0 \mathrm{~mm}$ is the same as at $L=2.2 \mathrm{~mm}$. However, all the spectra become softer for the larger target thickness. This is indicated already by the increase in $\varepsilon^{(1)}, \varepsilon^{(2)}$ values which are now $\varepsilon_{\mathrm{cr}}^{(1)} \simeq 85 \mathrm{MeV}, \quad \varepsilon_{\mathrm{cr}}^{(2)} \simeq 185 \mathrm{MeV}, \quad \varepsilon_{\mathrm{am}}^{(1)} \simeq 75 \mathrm{MeV}$, and $\varepsilon_{\mathrm{am}}^{(2)} \simeq 165 \mathrm{MeV}$. It is clear that the magnitude of the yield from the thicker target is essentially larger but this increase is different in the crystal and amorphous cases. For example, in the energy range $\varepsilon<45 \mathrm{MeV}$, the yield is increased by $6-7$ times for a crystal and by $17-$ 20 times for amorphous samples. As a result, the enhancement at $L=9.0 \mathrm{~mm}$ is almost 3 times less than at $L=2.2 \mathrm{~mm}$ in this energy range. At $L=9.0 \mathrm{~mm}$ the enhancement is peaked in the first bin $[\varepsilon \in$ (5-15) $\mathrm{MeV}$ ] for every collimation. Its maximum values are $\mu_{\text {max }}\left(\vartheta_{\text {out }} \leq 180^{\circ}\right) \simeq 2.25, \mu_{\text {max }}\left(\vartheta_{\text {out }} \leq 24^{\circ}\right) \simeq 2.15$, $\mu_{\text {max }}\left(\vartheta_{\text {out }} \leq 12^{\circ}\right) \simeq 2.08$, and $\mu_{\text {max }}\left(\vartheta_{\text {out }} \leq 1^{\circ}\right) \simeq 2.06$. The enhancement monotonically decreases with growing positron energy and approximately halves at $\varepsilon \approx$ $250 \mathrm{MeV}$. Thus, positron spectra from the crystal target are softer at $L=9.0 \mathrm{~mm}$ as well, and this property is much more pronounced in comparison with $L=2.2 \mathrm{~mm}$.

Matching systems can be characterized also by the maximum transverse momentum $p_{\perp}^{\max }$ of accepted positrons. In this connection, spectra of positrons having $p_{\perp}<p_{\perp}^{\max }$ are of undoubted interest. Such spectra at $L=$ $2.2 \mathrm{~mm} \mathrm{(a)} \mathrm{and} \mathrm{at} L=9.0 \mathrm{~mm}$ (b) from crystal and amorphous targets are shown in Fig. 4.

In contrast to the case of the pure angular selection (cf. Figs. 2 and 3), the position of spectral maxima at limited $p_{\perp}$ values is always in the first bin $[\varepsilon \in(7.5-12.5) \mathrm{MeV}]$. Corresponding maximum values are $\mu_{\max }(5 \mathrm{MeV} / c) \simeq$ 5.82, $\mu_{\max }(2.5 \mathrm{MeV} / c) \simeq 5.62$ at $L=2.2 \mathrm{~mm}$, and $\mu_{\max }(5 \mathrm{MeV} / c) \simeq 2.17, \mu_{\max }(2.5 \mathrm{MeV} / c) \simeq 2.11$ at $L=$ $9.0 \mathrm{~mm}$. The enhancement monotonically decreases with growing positron energy. Its variation over the whole energy interval presented in Fig. 4 is about $15 \%$ at $L=$ $2.2 \mathrm{~mm}$ and $40 \%$ at $L=9.0 \mathrm{~mm}$. So, for this selection too, positron spectra from crystal targets are softer than those from amorphous targets of the same thickness. The interesting feature of spectral curves in Fig. 4 is the similarity of those obtained for two different values of
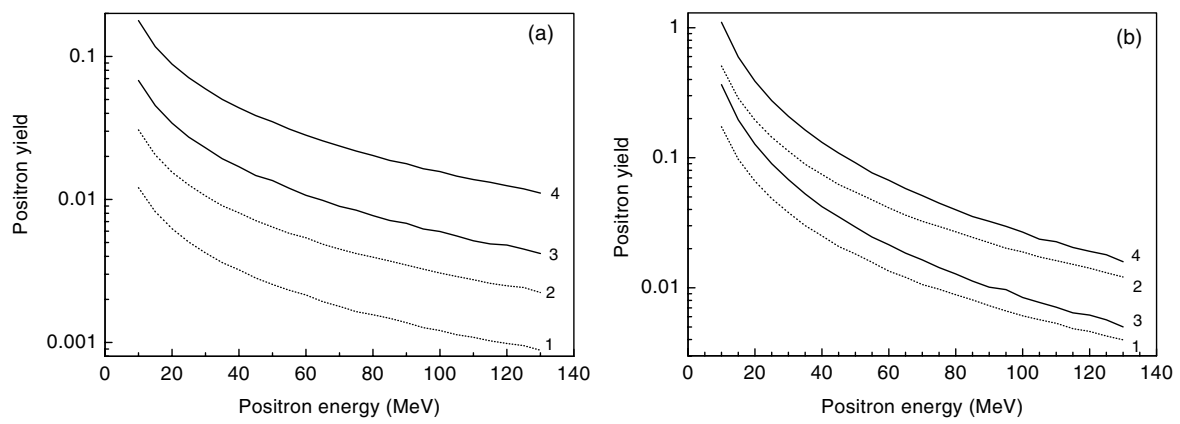

FIG. 4. Positron yield depending on energy at $L=2.2 \mathrm{~mm}$ (a) and $L=9.0 \mathrm{~mm}$ (b) for $p_{\perp}^{\max }=2.5 \mathrm{MeV} / c$ (curves 1 and 3) and for $p_{\perp}^{\max }=5 \mathrm{MeV} / c$ (curves 2 and 4). Solid curves represent the yield from crystal and dotted curves from amorphous targets. 

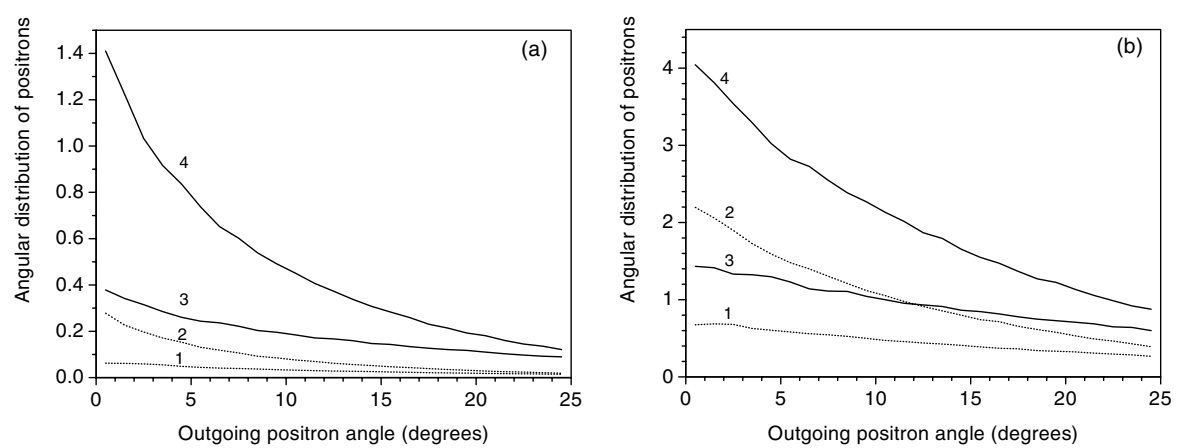

FIG. 5. Angular distribution $d N^{(+)} / d \Omega$ depending on outgoing positron angle at $L=$ $2.2 \mathrm{~mm}$ (a) and at $L=9.0 \mathrm{~mm}$ (b) for $p \in(8.5-11.5) \mathrm{Mev} / c$ (curves 1 and 3) and for $p \in$ (17-23) Mev/c (curves 2 and 4). Solid curves represent the yield from crystal and dotted curves from amorphous targets.

$p_{\perp}^{\max }$ from the same target. The scaling factors $\eta$ are $\eta_{\mathrm{cr}} \simeq$ $2.6, \eta_{\mathrm{am}} \simeq 2.5$ at $L=2.2 \mathrm{~mm}$ and $\eta_{\mathrm{cr}} \simeq 3.1, \eta_{\mathrm{am}} \simeq 3.0$ at $L=9.0 \mathrm{~mm}$. These factors turn out to be practically (within an accuracy of a few percent) independent of the total positron momentum $p$. This fact can be easily understood if we assume that the width of the angular distribution of positrons is completely due to multiple scattering being, thereby, proportional to $p^{-1}$. Such an assumption is confirmed by results of the calculation shown in Fig. 5 for two groups of positrons. One of them contains positrons having momentum in the interval $p \in(8.5-11.5) \mathrm{Mev} / c$, for another group $p \in$ (17-23) Mev/c.

For the given target, the width of the angular distribution of positrons with $p \approx 10 \mathrm{Mev} / c$ is approximately twice as much that for $p \approx 20 \mathrm{Mev} / c$ as expected. The width of every distribution evidently increases when we go on to the thicker target of the same kind. Comparing angular distributions from crystal and amorphous targets of the same thickness, we find that at $L=9.0 \mathrm{~mm}$ the distributions are somewhat (about $1.5^{\circ}$ ) wider in the crystal case for both groups. In units of FWHM of the distribution from the crystal target these differences are about $6.5 \%$ at $p \approx 10 \mathrm{Mev} / c$ and $14 \%$ at $p \approx$ $20 \mathrm{Mev} / c$. At $L=2.2 \mathrm{~mm}$ the distribution from the crystal target is wider by $15.5 \%$ at $p \approx 20 \mathrm{Mev} / c$ whereas this is narrower by $10 \%$ at $p \approx 10 \mathrm{Mev} / c$.

Going on to the comparison of our results with those obtained in [16], let us remember that to perform an accurate comparison of such kind, exact information is needed concerning the acceptance conditions and registration efficiency of detectors in the experiment. As noted in [16], at $p=20 \mathrm{Mev} / c$, the momentum acceptance $(\Delta p / p)$ was $3 \%$ (FWHM) and the polar angle acceptance was less than $20 \mathrm{mrad}$ (FWHM). Since the shape of the acceptance curves was unavailable to us, we have tried to simulate experimental conditions using the same angular collimation $\vartheta_{\text {out }} \leq \vartheta_{\text {out }}^{\max }$ and the same value of $\Delta p / p$ for all momenta and targets. So, at the calculation of the magnitudes of positron production efficiency (PPE), we simply put $\vartheta_{\text {out }}^{\max }$ to $20 \mathrm{mrad}$. The value of $\Delta p / p$ was chosen to reproduce at applied collimation the experimental magnitude of PPE for the 9.0-mm-thick amorphous target. Acting in this way, we have $\Delta p / p=3.2 \%$. We realize that our regard for the acceptance conditions is rather rough. An additional inaccuracy was introduced
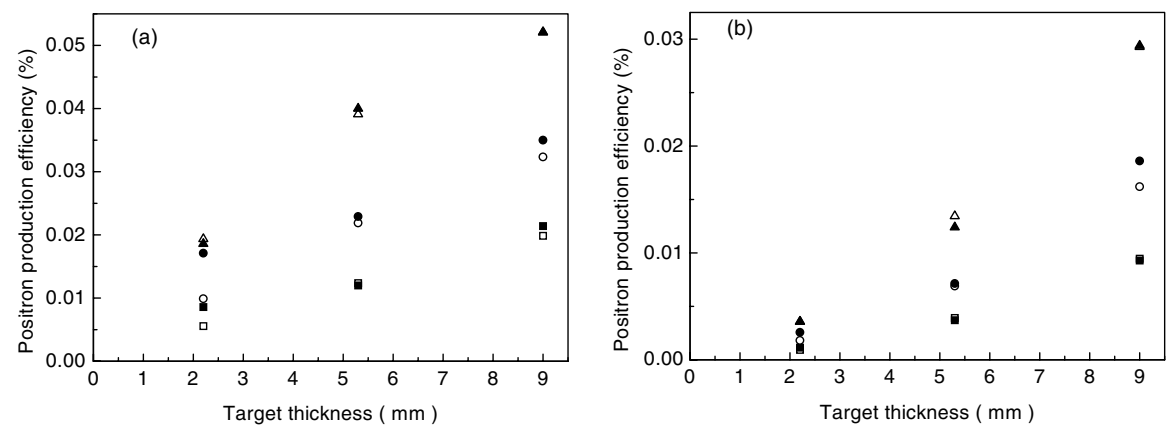

FIG. 6. Positron production efficiency from crystal (a) and amorphous (b) targets depending on thickness. Open symbols: our calculation; filled symbols: results from Fig. 5 of [16]; open triangles are for $p=20 \mathrm{Mev} / c$, open circles are for $p=15 \mathrm{Mev} / c$, and open squares are for $p=10 \mathrm{Mev} / c$. 
TABLE I. Enhancement of the positron yield from crystal targets.

\begin{tabular}{ccccccc}
\hline \hline \multirow{2}{*}{$\begin{array}{c}\text { Momentum } \\
(\mathrm{MeV} / \mathrm{c})\end{array}$} & \multicolumn{2}{c}{$\begin{array}{c}\text { Enhancement } \\
(2.2 \mathrm{~mm} \text { thick })\end{array}$} & \multicolumn{2}{c}{$\begin{array}{c}\text { Enhancement } \\
(5.3 \mathrm{~mm} \text { thick })\end{array}$} & \multicolumn{2}{c}{$\begin{array}{c}\text { Enhancement } \\
(9.0 \mathrm{~mm} \text { thick })\end{array}$} \\
\hline 10 & $6.0 \pm 0.5$ & $6.5 \pm 0.6$ & $3.2 \pm 0.3$ & $3.4 \pm 0.7$ & $2.1 \pm 0.2$ & $2.3 \pm 0.4$ \\
15 & $5.5 \pm 0.3$ & $6.2 \pm 0.8$ & $3.2 \pm 0.2$ & $3.2 \pm 0.5$ & $2.0 \pm 0.1$ & $2.0 \pm 0.2$ \\
20 & $5.4 \pm 0.2$ & $5.1 \pm 0.5$ & $2.9 \pm 0.1$ & $3.0 \pm 0.5$ & $1.8 \pm 0.1$ & $1.8 \pm 0.2$ \\
\hline \hline
\end{tabular}

when we determined the PPE numbers from Fig. 5 of [16]. Note that the experimental numbers obtained in such a way, which are presented by filled symbols in Fig. 6, do not reproduce exactly the whole set of mean experimental values for the enhancement given in Table I of [16]. Moreover, in Fig. 5 of [16] there are no experimental points for 2.2 and 5.3-mm-thick amorphous targets. For these two cases, we present in Fig. 6 the values of PPE given by smooth-curve fits corresponding to simulation fitting in Fig. 5 of [16]. Bearing all this in mind, we, nevertheless, can assert that a rather good agreement is seen in Fig. 6 of the experimental results and our estimations. A relative difference of them is better than $13 \%$ everywhere except the values of PPE at $p=10$ and $15 \mathrm{Mev} / c$ from both thinnest $(L=2.2 \mathrm{~mm})$ targets, where the experimental yield is underestimated by $19 \%$ to $42 \%$. Note that just for this thickness the largest inaccuracy was introduced while determining the PPE numbers from Fig. 5 of [16] at $p=10$ and $15 \mathrm{Mev} / c$, as the magnitude of the yield is especially small in this case.

In contrast to the magnitude of the positron yield, the enhancement is not very sensitive to the acceptance conditions. The calculated values of the enhancement (theory) are presented in Table I along with those taken from Table I of [16] (experiment). Purely statistical errors are figured in Table I as theoretical ones. The relative error in PPE was estimated as $N_{\mathrm{ef}}^{-1 / 2}$, where $N_{\mathrm{ef}}$ is the mean number of events in the phase space corresponding to the acceptance conditions used in calculations. The total statistics was chosen so that approximately to equalize values of $N_{\text {ef }}$ for amorphous and crystal targets of the same thickness. At given total statistics, the quantity $N_{\text {ef }}$ increases with growing positron momentum in accord with a shape of the positron spectra at hard collimation shown in Figs. 2 and 3. This fact leads to a better statistical accuracy for larger momentum. We emphasize that the differences of the estimated and experimental enhancement values are smaller than corresponding experimental errors for all momenta and samples figured in Table I.

\section{CONCLUSION}

Using the simple computer code suggested in [11,12], we have compared the theoretical predictions for some characteristics of the electromagnetic shower developing in axially aligned crystals with experimental results re- ported in $[13,14,15,16]$. On the whole, theory and experiment are consistent within the experimental accuracy. From this comparison we also conclude that the accuracy provided by the existing simplified code is at least better than $20 \%$. This accuracy may be slightly improved if we include into consideration some processes like annihilation of positrons or Compton scattering of photons which were ignored as corresponding cross sections are small in the energy region of interest. However, the approximate character of the radiation spectra at axial alignment used in our calculations still provides the main theoretical uncertainty. Nevertheless, we believe that the level of the accuracy already achieved in the theoretical description is quite sufficient to make a reliable choice for optimal parameters of the positron source using axially aligned single crystals.

\section{ACKNOWLEDGMENTS}

We are grateful to Professor H. Okuno for providing us with details of the experiment [16] and to the authors of [14] for numerous fruitful discussions. Support of this work by the Russian Fund of Basic Research under Grants No. 00-02-18007, No. 01-02-16926, and No. 01-02-22003 is also gratefully acknowledged.

[1] V. N. Baier, V. M. Katkov, and V. M. Strakhovenko, Phys. Status Solidi (b) 133, 583 (1986).

[2] R. Chehab et al., in Proceedings of the IEEE Particle Accelerator Conference, Chicago, 1989 (IEEE, Piscataway, NJ, 1989), pp. 283-285.

[3] V. N. Baier and R. Chehab, Positron Source based on Channeling Radiation, Proposal for an Experiment at Orsay, LAL, Orsay, France, 1990 (unpublished).

[4] V. N. Baier, V. M. Katkov, and V. M. Strakhovenko, Sov. Phys. Usp. 32, 972 (1989).

[5] V. N. Baier, V. M. Katkov, and V. M. Strakhovenko, Electromagnetic Processes at High Energies in Oriented Single Crystals (World Scientific, Singapore, 1998).

[6] V. N. Baier, V. M. Katkov, and V. M. Strakhovenko, Nucl. Instrum. Methods Phys. Res., Sect. A 250, 514 (1986).

[7] V. N. Baier, V. M. Katkov, and V. M. Strakhovenko, Nucl. Instrum. Methods Phys. Res., Sect. B 27, 360 (1987).

[8] R. Medenwaldt et al., Phys. Lett. B 227, 483 (1989).

[9] V. N. Baier, V. M. Katkov, and V. M. Strakhovenko, Nucl. Instrum. Methods Phys. Res., Sect. B 119, 131 (1996). 
[10] V. N. Baier, V. M. Katkov, and V. M. Strakhovenko, Phys. Status Solidi (b) 149, 403 (1988).

[11] V. N. Baier and V. M. Strakhovenko, Nucl. Instrum. Methods Phys. Res., Sect. B 155, 403 (1999).

[12] V. N. Baier, V. M. Katkov, and V. M. Strakhovenko, Nucl. Instrum. Methods Phys. Res., Sect. B 103, 147 (1995).

[13] R. Chehab et al., in Proceedings of the LINAC2000, Monterey, CA, 2000 (Stanford Linear Accelerator Center, Stanford, CA, 2000), p. 143.
[14] R.Chehab et al., in Proceedings of the RREPS-01 Conference, Lake Aya, Russia, 2001 [Nucl. Instrum. Methods Phys. Res., Sect. B (to be published)].

[15] M. Inoue et al., Nucl. Instrum. Methods Phys. Res., Sect. B 173, 104 (2001).

[16] H.Okuno et al., in Proceedings of the RREPS-01 Conference, Lake Aya, Russia, 2001 (Ref. [14]); KEK Report No. 2001-146, 2001. 